\title{
MILLIMETER RADIO EVIDENCE FOR CONTAINMENT MECHANISMS IN SOLAR FLARES
}

\author{
E. B. MAYFIELD, K. P. WHITE III, and F. I. SHIMABUKURO \\ Aerospace Corp., El Segundo, Calif., U.S.A.
}

\begin{abstract}
Recent theories of solar flares are reviewed with emphasis on the aspects of pre-flare heating. The heating evident at 3.3-mm wavelength is analyzed in the form of daily maps of the solar disk and synoptic maps compiled from the daily maps. It is found that isotherms defining antenna temperature enhancements of $340 \mathrm{~K}$ correspond in shape and location to facular areas reported by Waldmeier. Maximum enhancements occur over sunspots or near neutral lines of the longitudinal magnetic fields which indicates heating associated with chromospheric currents. These enhancements are correlated with flare importance number and are observed to increase during several days preceding flaring. This evidence for a containment mechanism in the chromosphere is collated with current theories of solar flares.
\end{abstract}

\section{Introduction}

Most of the published theories of solar flares have been based on the presumption that the magnetic field of an active region is the source of energy for a flare. Although the exact process of field annihilation is not certain, it is believed that the conversion occurs at the neutral line or sheet separating fields of the opposite polarity. The principal difficulty for these theories as Parker (1963) showed, is the excessive time required for the process to occur. Whereas flares are observed to reach flash phase in typically $10^{2}$ to $10^{3} \mathrm{~s}$, calculations yield times of $10^{5} \mathrm{~s}$ or longer to provide the $10^{32} \mathrm{erg}$ expected to be released by a major flare. Despite this difficulty, the premise is regarded as correct and subsequent work has examined various models to identify a rapid mode of field diffusion and annihilation. Recent theoretical work based on magnetohydrodynamic models has been successful in identifying processes of field annihilation which are sufficiently fast and compatible with experimental observations.

One of the first of these was that of Jaggi (1963) who considered three types of hydromagnetic instabilities in a plasma; namely, infinite conductivity, finite conductivity, and magnetic field gradient. His results, which were based on work by Furth et al. (1963), showed that only the finite conductivity case leads to an instability with growth times of the order of seconds or minutes. Finite conductivity instabilities in a plasma have been investigated in detail by Furth et al. (1963) for the case of a current sheet between fields of opposite polarity. For this model they find two unstable modes, a rippling and a tearing mode. Applied to conditions in the chromosphere, Jaggi showed that the rippling mode gave excessive times except for very short wavelength instabilities, but that the tearing mode was unstable for long wavelengths and gave typical times of $10^{2}$ to $10^{3} \mathrm{~s}$. One of the characteristics of this model is that the temperature of the current sheet should rise prior to the onset of the instability.

Petschek (1964) proposed standing magnetohydrodynamic shock waves in the neutral region separating fields of the opposite polarity as the mechanism for con- 
verting magnetic to plasma energy. In this model, which is based on the Sweet (1958) $\mathrm{X}$-type magnetic field configuration, the plasma and fields flow into the neutral region and the fields are annihilated by Alfvén waves. Assuming typical flare volume values of $10^{9} \mathrm{~cm}$ for the scale length, $500 \mathrm{G}$ average field and $2 \times 10^{11} \mathrm{~cm}^{-3}$ density, the annihilation time is $10^{2}$ to $10^{3} \mathrm{~s}$. The inflow velocity determines the rate of energy conversion and is insensitive to conductivity in the shock region, but a conductivity corresponding to a temperature of $10^{6} \mathrm{~K}$ is assumed for the initial condition. Based on this high initial value of temperature at the neutral point, heating should be observed prior to a flare.

Syrovatskii (1969) considered collisionless dissipation of magnetic energy in the neutral region separating fields of opposite polarity as the annihilation mechanism. In this model, currents in the neutral sheet can increase very rapidly, leading to plasma turbulence and magnetic field reconnection in times of the order of $10^{-1} \mathrm{~s}$. This also results in charged particle acceleration to very high energies observed to accompany flares. Part of this energy is dissipated in heating the chromosphere and corona and is responsible for the observed optical radiation. The onset of the instability in this model is controlled by conditions in the neutral region and turbulent resistance in the plasma becomes important when the ion thermal velocity exceeds the directed (current) electron velocity. This indicates, for chromospheric conditions, temperatures of about $10^{4} \mathrm{~K}$ prior to a flare.

Coppi and Friedland (1971) have proposed a model which does not require shock or Alfvén waves in the neutral field region for the dissipation of field energy. Flow velocities into the neutral sheet are subsonic but sufficiently large to generate strong electric fields and to initiate turbulent electrical resistivity. This results in field annihilation in the neutral region from a tearing mode instability. They assume that the lower corona is the region of flare onset and show that the model is very sensitive to temperature variations. Although they presume that initially temperatures in the neutral region are not high, values of $10^{5}-10^{6} \mathrm{~K}$ are indicated as typical for flare conditions. This value is shown to be reached in phases. When instability occurs, the dissipation of about $10^{32} \mathrm{erg}$ can be released in about $10^{2}$ to $10^{3} \mathrm{~s}$.

Experimental evidence in support of these theories has been previously reported by Mayfield et al. (1970). Results of observations at $3.3 \mathrm{~mm}$ wavelength showed enhanced emission associated with magnetic fields, especially near the neutral lines separating fields of the opposite polarity. For regions in which antenna temperatures were enhanced by more than about $8 \%$ over a quiet area temperature, importance class 2 or greater flares were observed to occur usually within about $24 \mathrm{~h}$. Although the results of the investigation were limited to larger flares during a short period, it verified heating associated with flaring regions and particularly at or near the neutral region of magnetic fields.

The present study is based on an analysis of extensive observations of solar emission at $3.3 \mathrm{~mm}$ wavelength obtained with the Aerospace radio telescope. These data cover the years 1967 through 1972 and, with a few missing periods, provide a continuous record of solar emission. Flare data for the same period, obtained from Solar- 
Geophysical Data published by the National Oceanic and Atmospheric Administration, have been compared with the radio data. These results show a close association between regions of enhanced $3.3 \mathrm{~mm}$ radio emission and flares and statistical evidence for flare occurrence proportional to enhancement for importance $1 \mathrm{~N}$ and $1 \mathrm{~B}$ flares. The heating at millimeter wavelengths is observed to occur about $24 \mathrm{~h}$ prior to flare onset and to be near the neutral magnetic field region.

\section{Observations}

The observations were made with the $15-\mathrm{ft}(4.57 \mathrm{~m})$ diameter antenna at Aerospace in El Segundo, Calif. Although the instrument has been described by Jacobs and King (1965) and by King et al. (1966), a brief description is given. The antenna is a Cassegrain in a polar mount with surface tolerance for $400 \mathrm{GHz}(0.75 \mathrm{~mm})$ operation. For this study it was operated at $90 \mathrm{GHz}(3.3 \mathrm{~mm})$. Beamwidth at half-power points is about $2.8^{\prime}$ and pointing accuracy is about $20^{\prime \prime}$. The antenna is controlled by means of an on-line computer which permits various tracking modes. For this investigation, the antenna was programmed to obtain a temperature matrix of $19 \times 19$ points centered on the disk. The angular separation of the readings was 99". Emission temperatures are normalized to an undisturbed region near the center of the disk which was selected from daily maps and the Fraunhofer Institut maps. By normalizing to a quiet region, problems of absolute calibration and atmospheric attenuation are avoided.

Observations near the limb are inaccurate because of the antenna beamwidth which limits the useful area to less than $0.7 \boldsymbol{R}_{\odot}$. Analytical methods to restore the data beyond this radius have not been successful.

Daily maps are made from the normalized temperature matrix by constructing isotherms of the antenna temperature at $2 \%$ increments for $4 \%$ or greater enhancement. A typical isothermal map is shown in Figure 1 for 30 June 1971. This shows two regions which are enhanced $7 \%$ and $10 \%$. These maxima are closely associated with magnetic field regions near sunspots at these locations. For presentation of longer term data, the daily maps are plotted in synoptic format to show a solar rotation. These are organized after Waldmeier (1972) so that comparison can be readily made with the photospheric maps of the Sun. Each solar rotation shows maps of the emission as it appeared near central meridian passage for latitudes to $\pm 50^{\circ}$. In addition to the date which is identified by a dot, the time at which maps were made is identified by the short vertical line. Crosses shown on the figure mark locations used for normalizing emission temperatures. Location and maximum enhancement for each region are shown by the filled circle and given by the number next to it in percent of enhancement. Values of the isotherms are also given.

Examples of comparisons of the radio synoptic maps with the Waldmeier heliographic maps of the photosphere are presented in Figures 2, 3,4 and 5. During these four solar rotations, one can see at a glance how well the $4 \%$ enhancement contours correspond to the extensions of the facular regions. Differences in shape and extent 


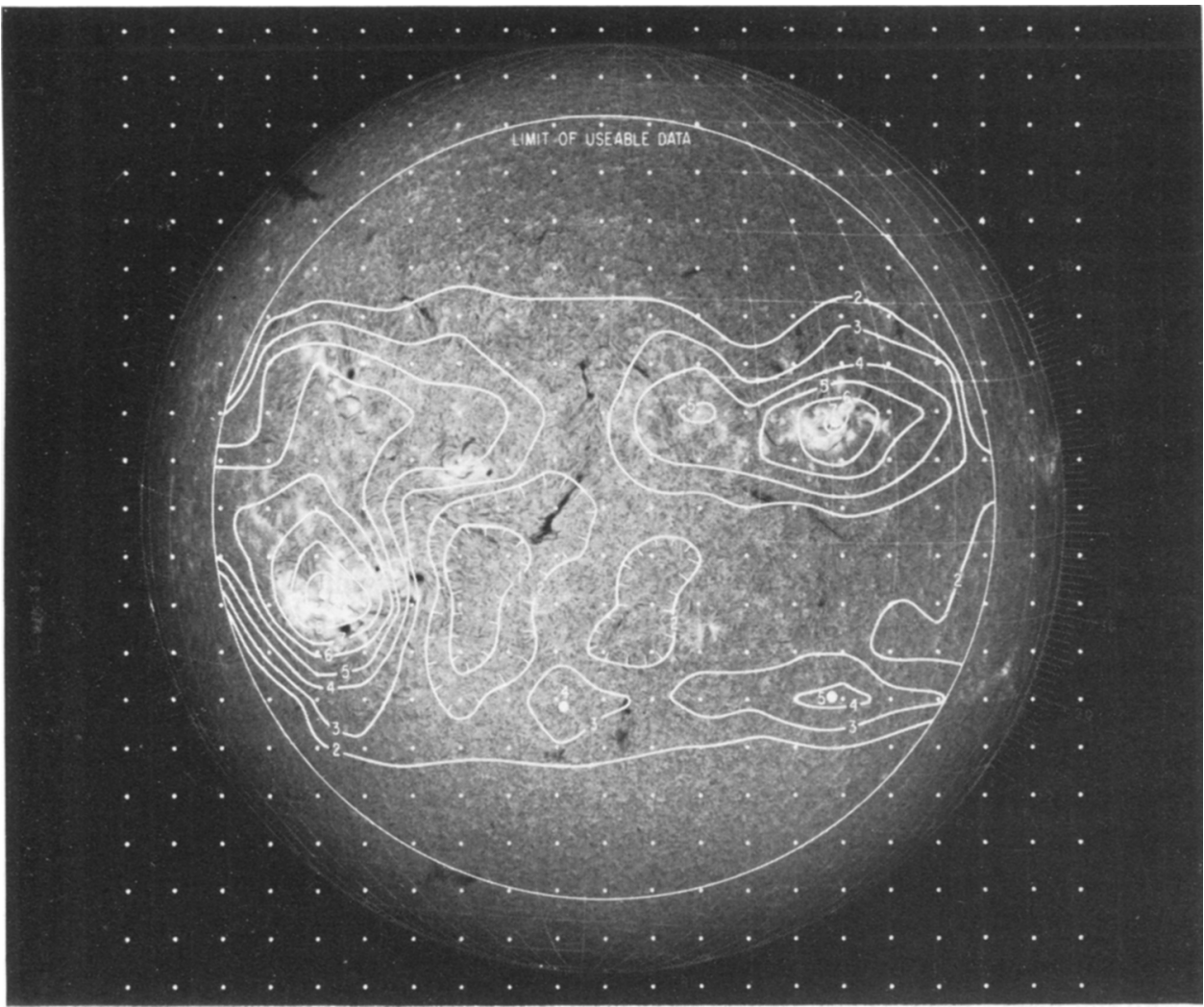

Fig. 1. The 3.3-mm solar radio temperature contour map made at The Aerospace Corp. on 30 June 1971 is superimposed on a concurrent $\mathrm{H} \alpha$ photograph. The contours are labeled in percent enhancement relative to the temperature of the undisturbed region denoted by the hatched contours.

can be attributed to a number of causes, which include: (1) slow, evolutionary changes in the active regions, since the photospheric maps pertain to the maximum development of the regions, while the millimeter maps reflect the appearance closest to central meridian passage; (2) rapid changes in the active regions, as caused by flares, whose residual effects can influence the radio contours (although this has been allowed for and corrected when possible); (3) selection of an invalid normalization point for a particular daily radio map (e.g., a dark absorption filament), which would cause an apparent 'growth' or 'contraction' of the enhancement levels, and; (4) the lack of a physical connection between the two phenomena.

The first three points are really just problems of observation and have been accounted for as described previously. The fourth point implies that, to the extent that the two phenomena are physically distinct, one would not expect a correspondence in shape and location. We feel that the striking correspondence evident in comparisons between the two types of maps for all of the solar rotations we have been able to analyze shows there is an apparent physical connection between the two phenomena. On the basis of these data, we will adopt the view that the facular regions and 


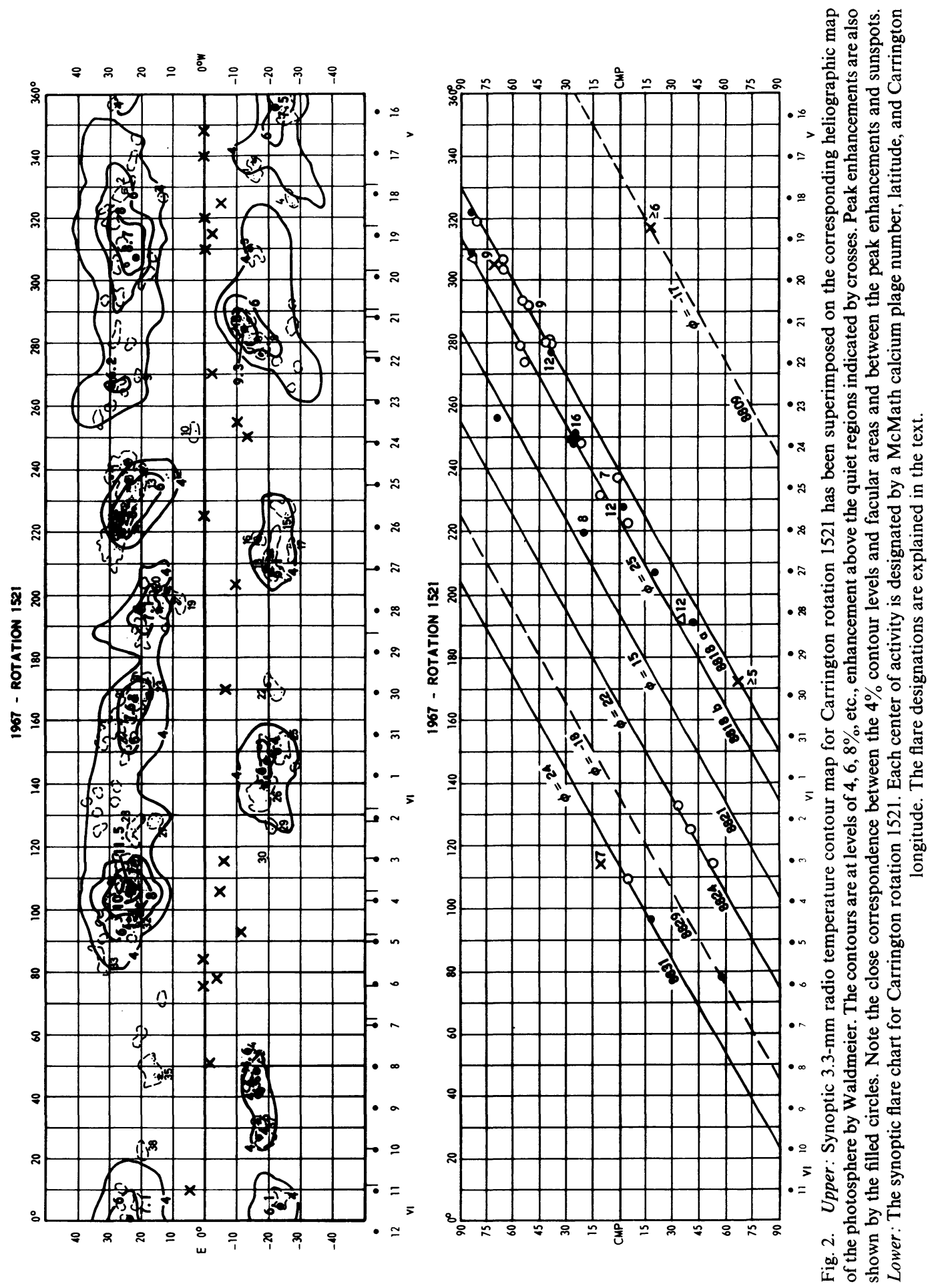




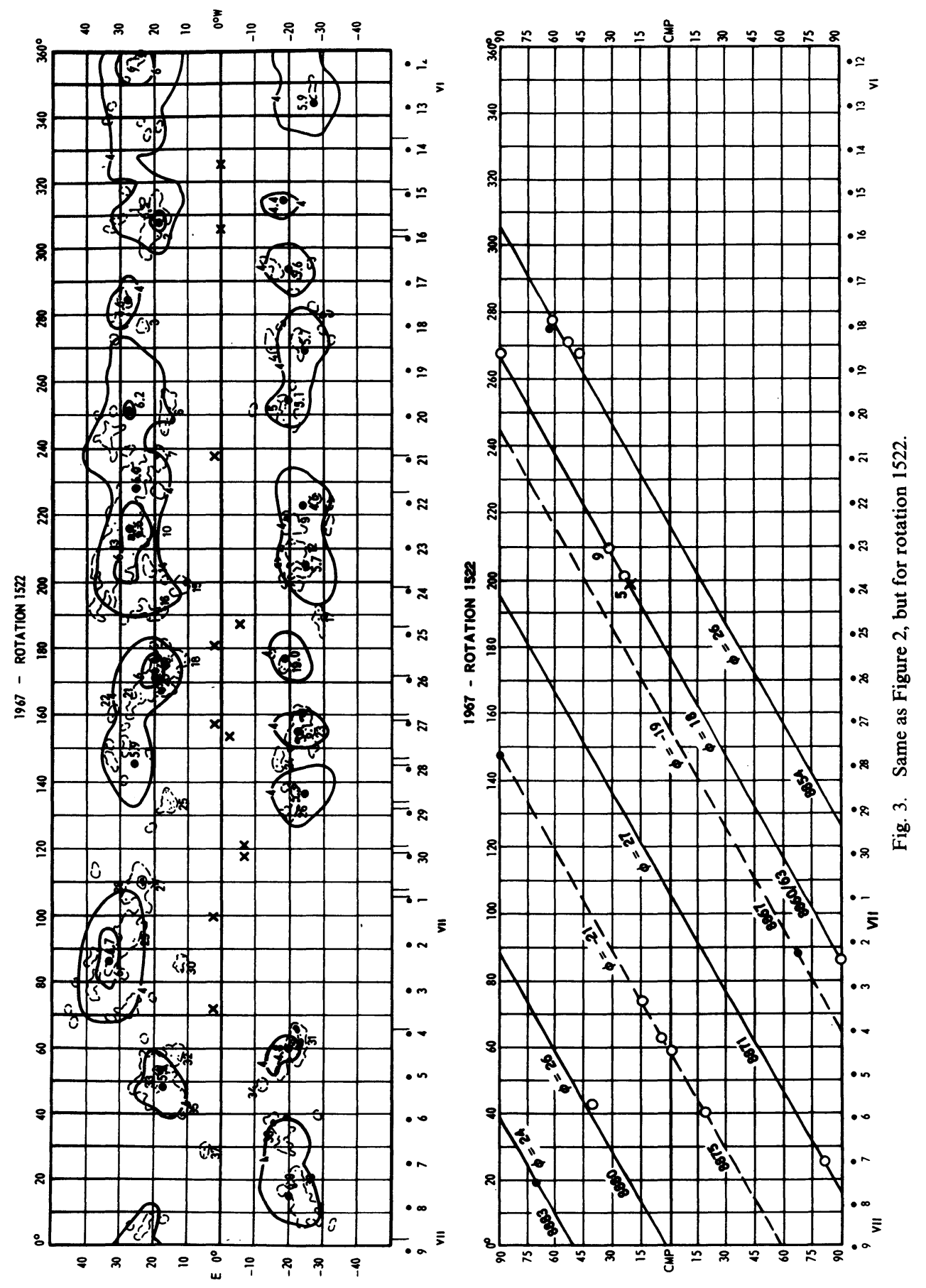




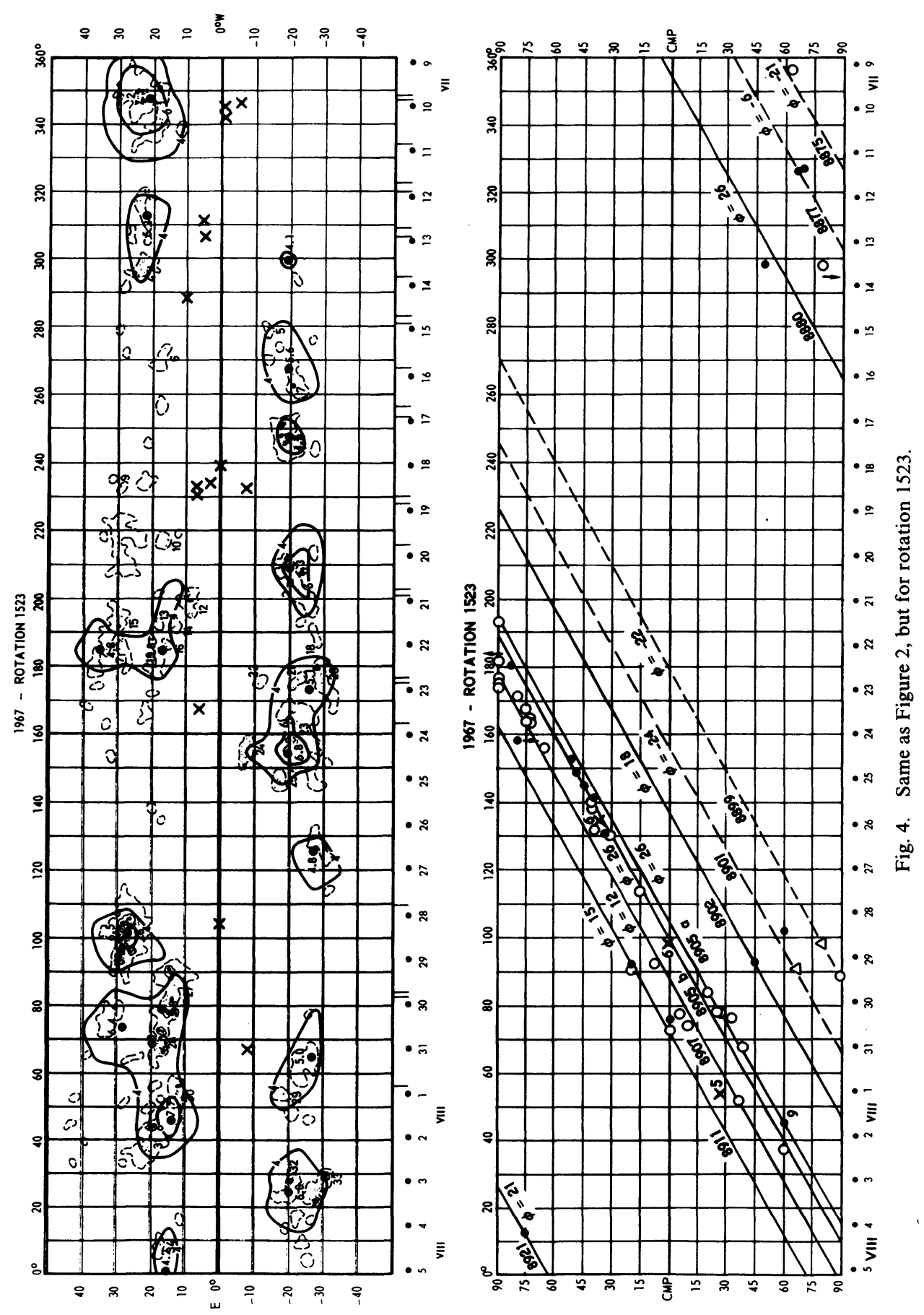


E. B. MAYFIELD ET AL.

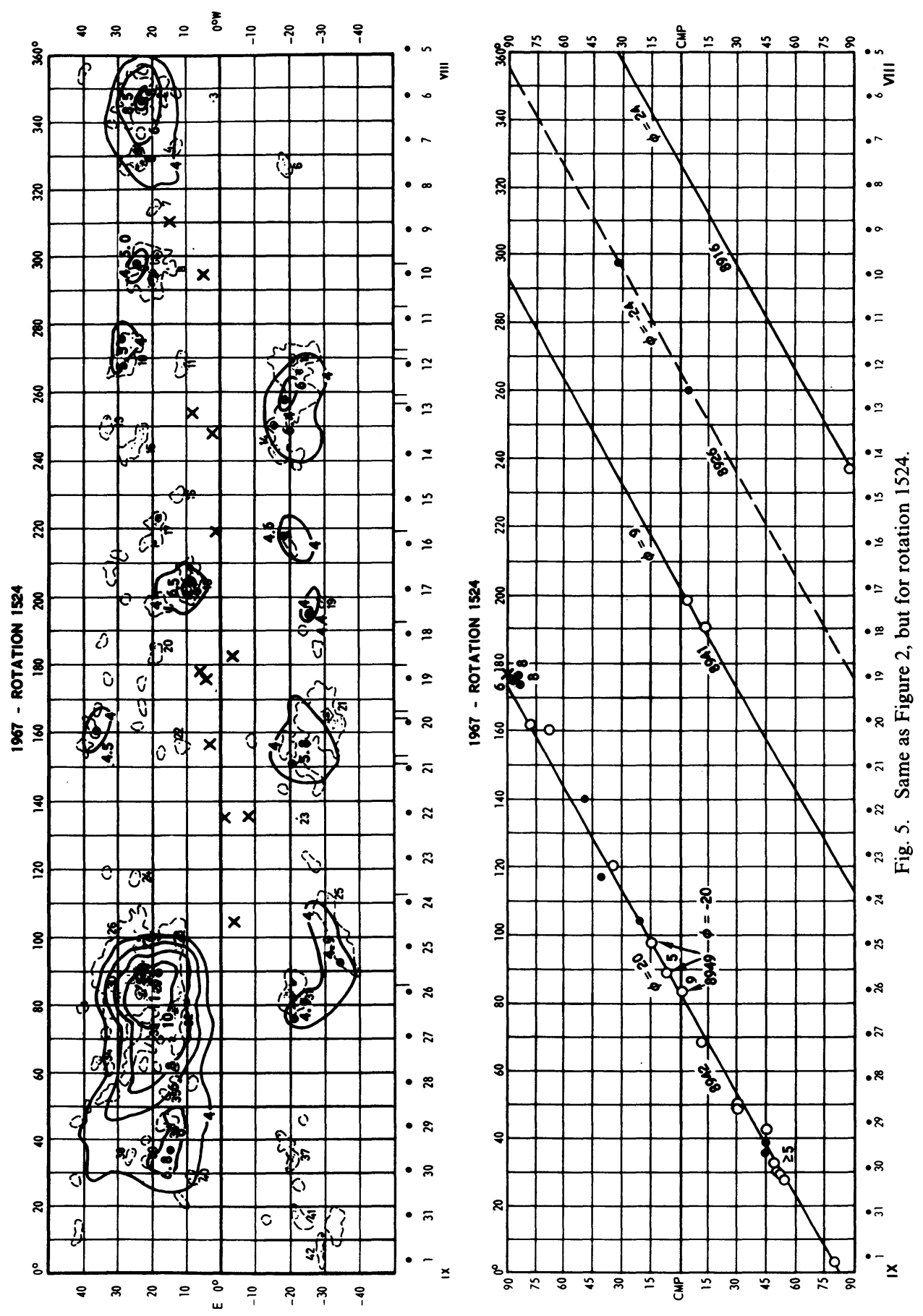


the millimeter enhancements are manifestations of the same phenomenon in the solar atmosphere; namely, magnetic fields which are responsible for the increased emissions in both cases. Figures 2 through 5 show that scattered facular regions, with no prominent sunspots, rarely exceed about $5 \%$ enhancement. If they do, the cause can usually be attributed to a scattering of small sunspots or pores, not all of which appear on Waldmeier's maps. Furthermore, the peak enhancements more likely than not, will correspond in location to the sunspots, even in active regions not exhibiting much flaring. These observations suggest regarding the measured millimeter enhancements as being made up of two components; one component can be attributed to heating which also manifests itself in the faculae; the other component should be associated with the sunspot magnetic fields and some sort of containment of an excessheated plasma. The latter component will be discussed in more detail in a later section dealing with flares. The former component will be discussed next.

By inspection, the contours in greatest agreement with the facular areas are the $4 \%$ contours. In some cases, a slightly greater contour, perhaps $5 \%$, would produce a cleaner correspondence. In either case, it is clear that most outlying facular regions, uncontaminated by the sunspot component, would lie between the $4 \%$ and $5 \%$ contours. Thus, we shall adopt a mean enhancement of $4.5 \% \pm 0.5$ as representative of the excess heating at $3.3 \mathrm{~mm}$ measured by the radio telescope. With the adoption of $7500 \mathrm{~K}$ (Linsky, 1973) as the temperature of the quiet sun, the enhancement above facular regions corresponds to about $340 \mathrm{~K}$. Such a measurement must be corrected for the fraction of the radio beam which is actually being filled by the heated structures. An estimate of such a fraction involves many assumptions about the fine structure of the low chromosphere and an indefensible extrapolation of models of heating in faculae, which models do not extend sufficiently high into the solar atmosphere. Future work could attempt to achieve consonance between the physical parameters indicated by the $\mathrm{mm}$ measurements and a facular model, such as that by Chapman (1970), but such is beyond the scope of the present work. For now the correspondence between the $4.5 \%$ enhancement at $3.3 \mathrm{~mm}$ and faculae can only be substantiated on the basis of spatial coincidence.

The question of the longevity of active regions can be investigated by inspection of successive synoptic maps to note the recurrence of given active regions. In this respect it is important to point out the sensitivity and precision of the data contained in the synoptic maps, because these parameters will directly influence the visibility of the enhancements. As stated before, the lowest contour level plotted corresponds to a $4 \%$ enhancement, so chosen because at this level individual active regions are usually discernable, whereas a $3 \%$ contour usually will run nearly the whole length of the map, providing very little information. Additionally, the repeatability of measurements made on different daily maps either on the same day or different days can be as large as $\pm 0.5 \%$, though the average is probably close to $\pm 0.3 \%$, with some outstanding examples showing variations of no more than $0.2 \%$ over a five or six-day period. To maintain a precision of about $10 \%$ (e.g. $4 \pm 0.4 \%$ ), we felt it was safest to display the $4 \%$ contour as the lowest level. 
Therefore, the longevity of an active region is defined as the time from when it first exceeded $4 \%$ enhancement to when it first disappeared below the $4 \%$ level. In this context, some regions are found to last less than a full solar rotation, while others can persist and be followed over five or six rotations. This result is entirely consistent with the result that the $4 \%$ contour level coincides in shape and location with the facular areas, which have lifetimes that can be as short as a few days or as long as a few solar rotations.

Another feature of the synoptic maps whose longevity can be gauged is the quiet regions, as indicated by the clustering of crosses. The nature of these regions is still in question, but certain examples of them can be followed over three or four rotations.

Solar flare data for the period 1967 through 1972 were plotted in synoptic format for comparison with the mm maps. Examples of these are shown in Figures 2-5 for rotations 1521-1524. The longitude and date are given at the bottom of the plots and central meridian distance is given vertically. This permits displaying all flares for an active region during a disk passage as well as its time of occurrence and location. Heliographic longitude for each region is determined from time of central meridian passage, and latitude as well as McMath-Hulbert calcium plage number is indicated with each region. These plots show a consistency for the flaring locations to remain fixed in position during a disk passage. In complex regions two or more centers may exist and these show only small excursions from a fixed location. Although the reports from individual observatories show significant dispersion in location and time, the mean values reported in the Comprehensive Part of Solar-Geophysical Data of the flare centroids are clearly very consistent.

The flares reported here are for importance number $1 \mathrm{~B}$ or greater. Those of number 1B are shown by open circles, importance 2 by filled circles and importance 3 by triangles. In addition we have included flares with an index number of 5 or greater based on the classification method of Dodson and Hedeman (1971). These are shown by crosses with the index number next to them. The Dodson-Hedeman classification method is based on five parameters: (1) X-ray emission, (2) $10 \mathrm{~cm}$ radio flux, (3) optical importance number, (4) meter wave flux and (5) dynamic radio spectrum.

\section{Discussion}

Active regions plotted in this synoptic fashion show a close association with a fixed location during a disk transit. Some regions have more than one active part but these typically remain distinct. These results show that flares are produced by unique areas of centers of activity which retain close association with the magnetic fields of the underlying sunspots. This is an important result and is in disagreement with the widely accepted opinion that flare data are subject to large errors and that both location and times of occurrence are very uncertain. Although this may be true for reports from individual observatories, the mean values reported in Solar-Geophysical Data are highly consistent.

Since flares occur in active regions near sunspot groups and the 3.3-mm emission 
is associated with the facular regions of magnetic fields, there is a correlation of the flares with enhanced $\mathrm{mm}$ regions. This correlation, however, is complex and no simple rules can be stated. Evidence for two components of the mm emission can be seen in the maps. One of these is associated with the general magnetic fields in the faculae and accounts for the enhancements of 4 or $5 \%$. This is the slowly-varying component and has been shown by Shimabukuro et al. (1973) to be due to magnetoionic processes in fields. The second component which accounts for the larger, rapidly-changing emission is associated with strong fields and occurs near the neutral lines or sheets of these fields. This strong emission is probably caused by currents in the neutral sheet separating fields of the opposite polarity as has been postulated by the theories discussed above.

Centers of activity which produce the greatest number of flares occur in regions of enhanced 3.3-mm emission, typically with enhancements greater than $8 \%$. A distinctive feature of the mm-emission in these centers is that the radio plage is usually small and the thermal gradient is large. The $4 \%$ isotherm tends to occur near the maximum of emission. In other centers which may have a large enhancement but produce few or small flares, the $4 \%$ isotherm encloses a large area. This indicates that major flareproducing regions have concentrated magnetic fields with necessarily large magnetic flux density and associated $\nabla \times H$ current densities. These results are consistent with previous reports that magnetically complex $\gamma$-type sunspot groups produce the greatest number of flares.

In the most active regions, the location of the flare centroids is usually near the maximum of the mm emission. Since the longitude of each active region is very stable during a single transit and the $\mathrm{mm}$ regions change slowly, the association of flare centroid and maximum enhancement is close. For less active regions, however, the flare center is frequently located several degrees from the maximum and may be as great as $10 \mathrm{deg}$. The $\mathrm{mm}$ enhancements in these cases are usually part of a complex region or two regions joined by a common $4 \%$ isotherm. This indicates that the stronger magnetic flux of the original sunspot group has been dissipated into the region marked by faculae and associated with the slowly-carying component of the $\mathrm{mm}$ emission. Location of the flare centers for these regions is usually within the 5 or $6 \%$ isotherm but rarely a flare may occur at the $4 \%$ level of the mm maps.

Although all of the centers of activity which flare are associated with an enhanced region of mm emission, the reverse is not true and a few regions with up to 8 or $9 \%$ enhancement may not flare. These are typically part of a large multiple region similar to those which produce a few small flares during a transit. They provide additional information on the kinetic processes in chromospheric magnetic fields which lead to flares and are valuable in evaluating flare theories.

On the basis of these results, it is not possible to distinguish critically between the proposed theoretical models of flares. The model of Furth et al. (1963) is specific, however, in discussing two instability modes with differing time constants, i.e. nonlocal and local. The pre-flare millimeter heating supports this model and indicates that the large-scale instability has a time constant of about $24 \mathrm{~h}$. Further the data 
show that a critical enhancement is reached at about $8 \%$ and for heating greater than that, a region will always produce a flare of importance $1 \mathrm{~N}$ or greater. This is shown in Figure 6 which summarizes the data for $1 \mathrm{~N}$ flares. Similar results are observed for 1B flares and also for importance 2 or greater flares as has been previously reported by Mayfield et al. (1970). This is also consistent with the Furth et al. model and indicates initiation of local instabilities although this does not exclude other models which require high temperatures as an initial condition to instability. Lack of antenna resolution prevents indentification of precise locations of the millimeter heating in the neutral magnetic field region which could distinguish smaller and hotter areas in this

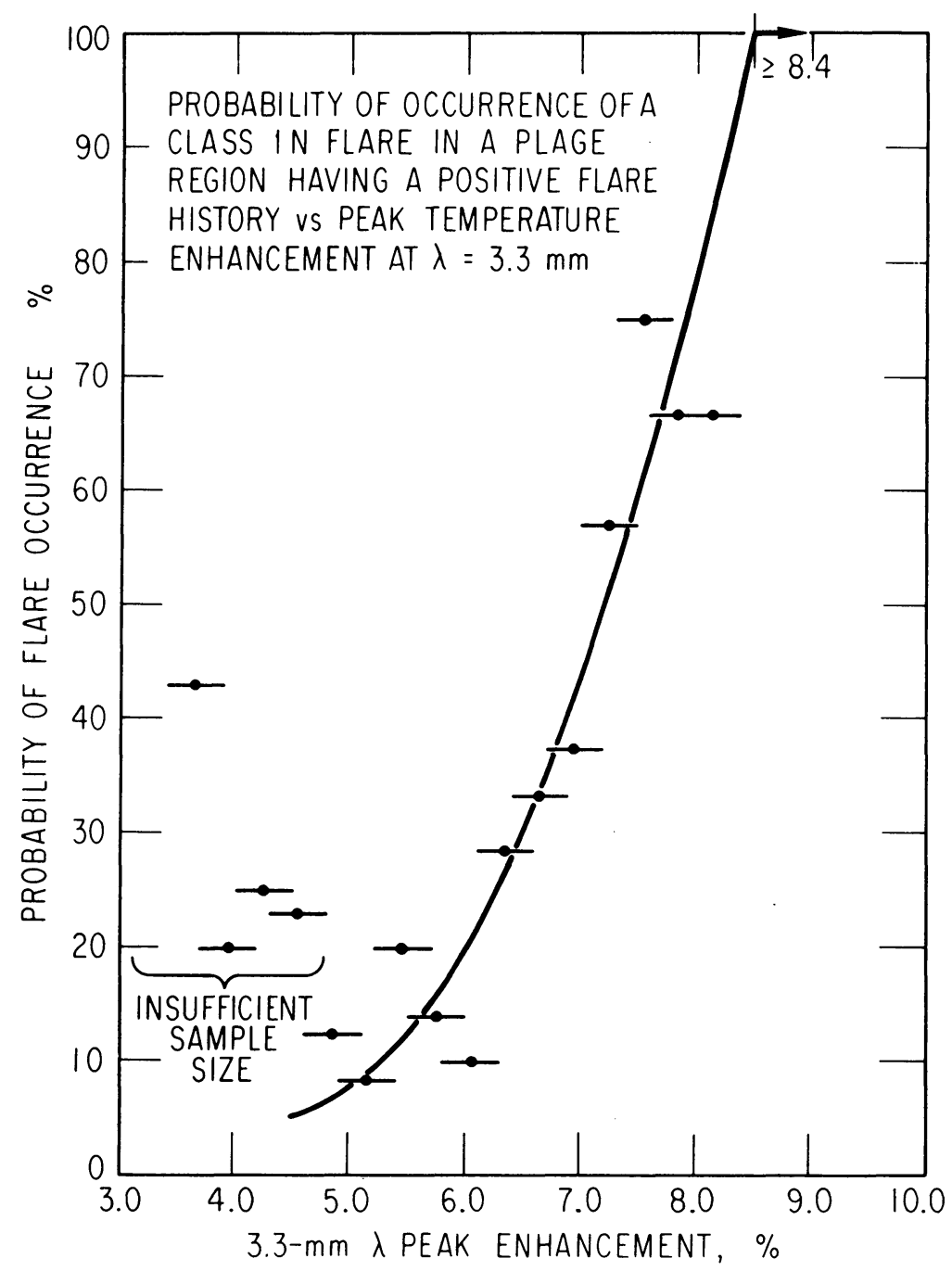

Fig. 6. The probability of occurrence of a class $1 \mathrm{~N}$ flare-prone plage region within one to two days following measurement of the peak temperature enhancement at 3.3-mm wavelength, given as a function of the peak temperature enhancement. 
transition. No apparent changes have been observed in either $\mathrm{H} \alpha$ filtergrams or magnetograms of the longitudinal component of the field associated with the $8 \%$ enhancement of the $3.3 \mathrm{~mm}$ emission.

\section{Concluding Remarks}

These extensive observations of solar emission at $3.3 \mathrm{~mm}$ wavelength show that strong enhanced regions are closely associated with the neutral region of longitudinal magnetic fields. The $4 \%$ enhanced isotherms agree closely with the facular regions and indicate that this is also due to magnetic fields. These features of the emission have been shown to be consistent with magneto-ionic theory. Regions which are enhanced greater than $8 \%$ always flare and indicate that some mechanism critical to flares occurs at this level. These results are consistent with recent hydromagnetic theories of flares which are based on magnetic field annihilation at the neutral region of opposing polarities. The observed heating is presumed caused by a current sheet in the neutral region.

\section{Acknowledgements}

The authors thank Mr P. Alailima who helped obtain the radio data and Mrs Mary Gates who helped in preparing the synoptic maps.

Part of this work was supported by NASA under contract NAS2-7292, part of it by Air Force Contract F04701-72-C-0073 and part of it by the Aerospace Company Financed Research Program.

\section{References}

Chapman, G. A. : 1970, Solar Phys. 14, 315.

Coppi, B. and Friedland, A. B.: 1971, Astrophys. J. 169, 379.

Dodson, H. W. and Hedeman, E. R.: 1971, NOAA Report UAG-14, World Data Center A, U.S. Dept. of Commerce.

Furth, H. P., Killeen, J., and Rosenbluth, M. N.: 1963, Phys. Fluids 6, 459.

Jacobs, E. and King, H. E.: 1965, I.E.E.E. International Convention Record Part 5.

Jaggi, R. K.: 1963, J. Geophys. Res. 68, 4429.

King, H. E., Jacobs, E., and Stacey, J. M.: 1966, I.E.E.E. Trans. on Antennas and Propagation, A.P. $14,82$. Linsky, J. L. : 1973, Solar Phys. 28, 409.

Mayfield, E. B., Higman, J., and Samson, C.: 1970, Solar Phys. 13, 372.

Parker, E. N.: 1963, Astrophys. J. Suppl. 8, 177.

Petschek, H. E.: 1964, in W. N. Hess (ed.), AAS-NASA Symposium on the Physics of Solar Flares, Government Printing Office, Washington, D.C., p. 425.

Shimabukuro, F. I., Chapman, G. A., Mayfield, E. B., and Edelson, S. : 1973, Solar Phys. $30,163$.

Solar-Geophysical Data: 1967-1972, U.S. Dept. of Commerce, Boulder, Colo.

Sweet, P. A.: 1958, in B. Lehnert (ed.), 'Electromagnetic Phenomena in Cosmical Physics', IAU Symp. 6, 123.

Syrovatskii, S. I. : 1969, in C. de Jager and Z. Śvestka (eds.), 'Solar Flares and Space Research', COSPAR Symp., 346.

Waldmaier, M.: 1972, 'Heliographic Maps of the Photosphere', Publikationen der eidgenössischen Sternwarte Zürich.

\section{DISCUSSION}

Smith: You have used the word containment. Isn't it possible that the heating is due to slow continuous reconnection at a current sheet? 
Mayfield: We have used the word containment because there is a threshold beyond which a region is certain to flare.

Smith: This is confusing terminology because most people interpret containment as containment of particles. What you are really talking about is a threshhold.

Mayfield: We interpret the enhanced $3.3 \mathrm{~mm}$ radio emission preceding flares as evidence for containment because of the existence of a critical level of enhancement. This does not show the presence of energetic particles but does indicate a heating mechanism that is a precursor to flares and is observed to occur about $24 \mathrm{~h}$ prior to onset.

Kundu: (1) I would like to point out that we have shown conclusively that $\mathrm{H} \alpha$ dark filaments and limb prominences are associated with absorption and emission features at $3 \mathrm{~mm}$. In your first slide where you associate a $3 \mathrm{~mm}$ depression with a coronal hole, I believe I've seen a fairly large filament whose orientation is in general agreement with the orientation of your depression feature. Consequently I feel that it might as well be associated with the filament.

(2) I do not understand why you are associating the $3 \mathrm{~mm}$ emissive regions with the magnetic neutral line only. As you know, these regions are polarized - the emission comes from two regions of opposite polarity, separated by a line of zero polarity or of neutral line as seen by Mt. Wilson magnetograms.

(3) Regarding the preheating as an indicator of flare production, I would like to make the following comment. The phenomenon of preheating was first observed by the French group in 1957 . We noticed that several hours before a burst occurs a region greatly increases in intensity and polarization within a coresize of about $1^{\prime}$ at $3 \mathrm{~cm}$. Indeed if we plot the number of bursts as a function of the core-intensity of the region, we find a very similar linear relation to that indicated in your last slide at $3 \mathrm{~mm}$. Consequently, I contend that the $3 \mathrm{~cm}$ preheating can be used as efficiently as an indicator of flare production as your $3 \mathrm{~mm}$ preheating. This result was published in Compt. Rend. Acad. Sci. 1957, Ann. Astrophys. 22, Paris Symposium on Radio Astronomy and in Solar Radio Astronomy.

Mayfield: (1) The data we report have a resolution of only $2.8^{\prime}$ and we are not able to resolve filaments. We are aware of your observations which show the association of dark filaments and reduced emission. Our evidence for the association of reduced emission and coronal holes is not shown in Figure 1 but in later data and is still tentative.

(2) The polarized $\mathrm{mm}$ emission in a bipolar magnetic field has been observed to be right circular above the positive field and left circular above the negative field. However, the total power which we are reporting here is associated with the neutral line or region as shown in longitudinal magnetograms.

(3) Similar observations of enhancement and polarization prior to flares have been reported at 3 and $10 \mathrm{~cm}$ by others (particularly the Japanese). However our observations at $3.3 \mathrm{~mm}$ occur low in the chromosphere and show evidence of a critical temperature enhancement above which flares will occur within about $24 \mathrm{~h}$. 